Numer. Math. 75: 339-356 (1997)

Numerische Mathematik

(c) Springer-Verlag 1997

Electronic Edition

\title{
Galerkin and weighted Galerkin methods for a forward-backward heat equation
}

\author{
Hao $\mathrm{Lu}^{\star}$ \\ Department of Mathematics, University of Nijmegen, Toernooiveld, 6525 ED Nijmegen, \\ The Netherlands; e-mail na.hlu@na-net.ornl.gov
}

Received March 3, 1995

Summary. Galerkin and weighted Galerkin methods are proposed for the numerical solution of parabolic partial differential equations where the diffusion coefficient takes different signs. The approach is based on a simultaneous discretization of space and time variables by using continuous finite element methods. Under some simple assumptions, error estimates and some numerical results for both Galerkin and weighted Galerkin methods are presented. Comparisons with the previous methods show that new methods not only can be used to solve a wider class of equations but also require less regularity for the solution and need fewer computations.

Mathematics Subject Classification (1991): 65N30, 35K20

\section{Introduction}

In this paper we consider Galerkin and weighted Galerkin methods for the following forward-backward heat equation:

$$
\begin{gathered}
\sigma(x, t) u_{t}-u_{x x}=f(x, t), \quad \forall(x, t) \in \Omega=(-1,1) \times(0,1), \\
\begin{cases}u( \pm 1, t)=0 & \forall t \in(0,1), \\
u(x, 0)=0 & \text { for } \sigma(x, 0)>0, \\
u(x, 1)=0 & \text { for } \sigma(x, 1)<0,\end{cases}
\end{gathered}
$$

where the coefficient $\sigma(x, t)$ changes sign in $\Omega$.

Problem (1), (2) arises in various applications, such as, boundary layer problems in fluid dynamics (Stewartson $(1974,1981)$ ), plasma physics and astrophysics in the study of propagation of an electron beam through the solar corona (LaRosa (1986)).

\footnotetext{
* This work was partially supported by the Netherlands Organization for Scientific Research under grant 611-302-025
} 
Problems like $\sigma(x, t) u_{t}-u_{x x}=f(x, t)$ with $\sigma(x, t)$ taking different signs were first considered by Gevrey $(1913,1914)$. He treated in particular the case $\sigma(x, t)=x^{m}$ with $m$ an odd integer. Later, Baouendi and Grisvard (1968) dealt with the case $\sigma(x, t)=x$ in detail. A similar treatment in a context where the second-order derivative is replaced by a suitable nonlinear differential operator can be found in Lions (1969). Franklin and Rodemich (1968) considered also the case $\sigma(x, t)=x$ and treated the equation on $-\infty<x<\infty, 0<t<T$. Recently, $\mathrm{Lu}$ and Wen (1994) showed the existence and uniqueness of a weak solution for the equation (1), (2) on a certain Hilbert space. The existence and uniqueness of a weak solution in a different sense for a special $\sigma$ satisfying $\sigma \operatorname{sgn} x>0$ was given by Pagani (1976). The stability criterion in Lu and Wen (1994) shows the problem is well-posed.

It is well known that a common approach for a heat equation is first to apply the Galerkin method in space to reduce the equation to a set of ordinary differential equations. Then a suitable method is applied to integrate the ordinary differential equations. However, the forward-backward heat equation (1), (2) does not fit this category because the diffusion coefficient $\sigma(x, t)$ changes sign. Vanaja and Kellogg (1990) presented some iterative methods to solve finite difference approximation to (1), (2), in which the unknowns are swept in the order suggested by the equation, if $\sigma(x, t)=\sigma(x)$ or $\sigma_{t}(x, t) \leq 0$. Aziz and Liu (1991) consider a finite element method for (1) subject to

$$
\begin{cases}u(-1, t)=u(1, t)=0 & \forall t \in(0,1), \\ u(x, 0)=0 & \forall x \in(0,1), \\ u(x, 1)=0 & \forall x \in(-1,0) .\end{cases}
$$

Though it was pointed out that the problem (1), (3) is overdetermined in general (see Lu (1995)). Aziz-Liu's method can be used to solve (1), (2) with a straightforward modification under certain assumptions as mentioned in section 4 . Their approaches are to transform the equation to a first-order system of symmetricpositive partial differential equations in the sense of Friedrichs (1958) and solve the system by a finite element method.

The aim of the paper is to present Galerkin and weighted Galerkin methods for (1), (2) without transforming the equation to a first-order system of partial differential equations. We consider a simultaneous discretization of space and time variables by using continuous finite element methods. If there exist two functions $g, q \in H^{1}(\Omega)$ such that $|q| \leq C<+\infty$ and

$$
{ }_{2}^{1} g_{x}-\frac{1}{2} \sigma_{t}-\sigma q_{t}-q_{x}^{2} \geq \frac{\beta}{4} g^{2},
$$

which holds in particular for $\sigma_{t}(x, t) \leq b<\pi^{2} / 2$, our results show that the $L^{2}$ rate of convergence is $O\left(h^{k}\right)$, where $h$ is the meshsize of space and time, if the solution $u \in H^{k+1}(\Omega)$ and piecewise polynomials of degree $k$ are used.

It is shown also that the Galerkin method works well under certain assumptions even if $\sigma_{t}(x, t) \geq \pi^{2} / 2$ in some points of $\Omega$, but there exist some $\sigma(x, t)$ 
for which the Galerkin method may fail to solve (1), (2). The weighted Galerkin method can be useful to solve a wide class of the forward-backward heat equations like $\sigma(x, t) u_{t}-u_{x x}=f(x, t)$. Some examples show that for some $\sigma(x, t)$ the weighted Galerkin method can be used to solve (1), (2) efficiently in case the Galerkin method fails. The linear systems of the discrete equations arising from the methods are positive definite. Comparisons with previous methods known for the forward-backward heat equation, for example Vanja-Kellogg's method (1990) and Aziz-Liu's method (1991), show that the methods presented in this paper have the following advantages:

A. New methods can be used to solve a much wider class of equations than Vanaja-Kellogg's method and Aziz-Liu's method. The assumptions made on the coefficients of the equation is weaker than previous ones.

1. Aziz-Liu's assumptions are stronger than (4) if their method is used to solve (1), (2). Their assumptions imply (4). Furthermore, it is shown that if Aziz and Liu's method is applicable to (1), (2), so is the weighted Galerkin method.

2. The difference between $\sigma_{t}<\pi^{2} / 2$ and Vanaja-Kellogg's assumption $\sigma_{t} \leq$ 0 is essential. An example shows that doing a transformation $y=y(t)$ for a wide class of equations (1), (2) with $\sigma_{t} \geq \pi^{2} / 2$ for some points in $\Omega$, we obtain new forward-backward heat equations (1), (2) such that the corresponding $\sigma$ satisfies $\sigma_{t}<\pi^{2} / 2$, but there is no transformation $y=y(t)$ such that the corresponding $\sigma$ satisfies $\sigma_{t} \leq 0$.

B. New methods require less regularity for the solution of the equation than the previous numerical methods. Vanaja-Kellogg's method requires that the solution possesses continuous derivative of order 4 in $x$ and order 2 in $t$ to obtain the rate of convergence $O\left(k+h^{2}\right)$, where $k$ and $h$ are meshsize in time and in space, respectively. Aziz-Liu's method requires the solution $u_{t}, u_{t x}, u_{x x} \in L^{2}(\Omega)$ if it is used to solve (1), (2). The new methods need only the solution $u \in H^{1}(\Omega)$.

C. Both Galerkin and weighted Galerkin methods need fewer computations than Aziz-Liu's method. Though the new methods and Aziz-Liu's method share the same rate of convergence in theory, the numerical example shows that new methods by using a basis of piecewise linear functions achieve at least the same rate of convergence as Aziz and Liu method by using a basis of piecewise bivariate polynomials with degree $\leq 2$.

At the end of the introduction we introduce some notation to be used in the paper. Denote the boundary $\partial \Omega$ by $\Gamma_{1} \cup \cdots \cup \Gamma_{6}$, where $\Gamma_{i}$ are defined as follows:

$$
\begin{aligned}
& \Gamma_{1}=\{(x, t): x \in(-1,1), t=0, \sigma(x, 0) \leq 0\}, \\
& \Gamma_{2}=\{(x, t): x=-1, t \in(0,1)\}, \\
& \Gamma_{3}=\{(x, t): x \in(-1,1), t=1, \sigma(x, 1)<0\}, \\
& \Gamma_{4}=\{(x, t): x \in(-1,1), t=1, \sigma(x, 1) \geq 0\}, \\
& \Gamma_{5}=\{(x, t): x=1, t \in(0,1)\},
\end{aligned}
$$




$$
\Gamma_{6}=\{(x, t): x \in(-1,1), t=0, \sigma(x, 0)>0\},
$$

and the outward unit vector normal to $\partial \Omega$ by $n=\left(n_{x}, n_{t}\right)$.

Let $L^{2}(\Omega)$ be the standard space of square integrable functions on $\Omega$ with inner product $(\cdot, \cdot)$ defined by $(u, v)=\int_{\Omega} u v d \Omega$ and norm $\|u\|_{0}=(u, u)^{\frac{1}{2}}$. We use also the classical Sobolev space $H^{m}(\Omega)$ provided with the norm

$$
\|u\|_{m}=\left(\sum_{|\alpha| \leq m} \int_{\Omega}\left|\partial^{\alpha} u\right|^{2} d \Omega\right)^{\frac{1}{2}}
$$

and the seminorm

$$
|u|_{m}=\left(\sum_{|\alpha|=m} \int_{\Omega}\left|\partial^{\alpha} u\right|^{2} d \Omega\right)^{\frac{1}{2}} .
$$

Finally, define the test and trial space by

$$
V=\left\{v \in H^{1}(\Omega): v=0 \text { at } \Gamma \equiv \Gamma_{2} \cup \Gamma_{3} \cup \Gamma_{5} \cup \Gamma_{6}\right\} .
$$

and for $u \in H^{1}(\Omega)$ define

$$
\|u\|_{x}=\left(\|u\|_{0}^{2}+\left\|u_{x}\right\|_{0}^{2}\right)^{1 / 2}, \quad\|u\|_{x, w}=\|w u\|_{x},
$$

where $w \in H^{1}(\Omega)$ is a positive function.

\section{A Galerkin variational formulation}

Define $a(u, v)=\left(\sigma u_{t}, v\right)+\left(u_{x}, v_{x}\right)$. The Galerkin variational formulation of equation (1), (2) for a given $f \in L^{2}(\Omega)$ is to find a $u \in V$ such that

$$
a(u, v)=(f, v), \quad \forall v \in V,
$$

Note that for any $u \in H^{2}(\Omega) \cap V$ it is readily seen that

$$
a(u, v)=\left(\sigma u_{t}, v\right)-\left(u_{x x}, v\right) .
$$

Theorem 2.1 If there exists a function $g \in H^{1}(\Omega)$ such that

$$
g_{x}-\sigma_{t} \geq \frac{\beta}{2} g^{2}
$$

with $\beta>1$, then there exists a positive constant $C$ such that

$$
\|u\|_{x}^{2} \leq C a(u, u), \quad \forall u \in V .
$$


Proof. Friedrichs' first inequality shows that there exists a positive constant $\gamma$ such that

$$
\int_{\Omega} u_{x}^{2} d \Omega \geq \gamma\|u\|_{0}^{2}, \quad \forall u \in V
$$

which implies that (7) holds if and only if there exists a positive $\widetilde{C}$ such that

$$
\int_{\Omega} u_{x}^{2} d \Omega \leq \widetilde{C} a(u, u) .
$$

On the other hand, a simple computation shows that

$$
\begin{aligned}
a(u, u) & =\int_{\Omega}\left(\sigma u_{t} u+u_{x}^{2}-g u_{x} u+g u_{x} u\right) d \Omega \\
& =\int_{\Omega}\left(\frac{1}{2}\left(\sigma u^{2}\right)_{t}-\frac{1}{2} \sigma_{t} u^{2}+u_{x}^{2}-\frac{1}{2}\left(g u^{2}\right)_{x}+\frac{1}{2} g_{x} u^{2}+g u_{x} u\right) d \Omega \\
& =\int_{\Omega}\left(\frac{1}{2}\left(g_{x}-\sigma_{t}\right) u^{2}+g u_{x} u+u_{x}^{2}\right) d \Omega+\int_{\Omega}\left(\frac{1}{2}\left(\sigma u^{2}\right)_{t}-\frac{1}{2}\left(g u^{2}\right)_{x}\right) d \Omega .
\end{aligned}
$$

With the use of Green's formula, the last integration turns out to be

$$
\begin{aligned}
& \int_{\Omega}\left(\frac{1}{2}\left(\sigma u^{2}\right)_{t}-\frac{1}{2}\left(g u^{2}\right)_{x}\right) d \Omega=\int_{\partial \Omega}\left(\frac{1}{2} \sigma n_{t} u^{2}-\frac{1}{2} g n_{x} u^{2}\right) d S \\
= & \int_{\Gamma_{1} \cup \Gamma_{4}}\left(\frac{1}{2} \sigma n_{t} u^{2}-\frac{1}{2} g n_{x} u^{2}\right) d S=\int_{\Gamma_{1} \cup \Gamma_{4}} \frac{1}{2} \sigma n_{t} u^{2} d S \geq 0 .
\end{aligned}
$$

Hence, assuming that $g$ is a function satisfying (6), we have

$$
\begin{aligned}
a(u, u) & \geq \int_{\Omega}\left(\frac{\beta}{4} g^{2} u^{2}+g u_{x} u+u_{x}^{2}\right) d \Omega \\
& =\int_{\Omega}\left(\beta\left(g u / 2+u_{x} / \beta\right)^{2}+(1-1 / \beta) u_{x}^{2}\right) d \Omega \\
& \geq \widetilde{C}^{-1} \int_{\Omega} u_{x}^{2} d \Omega,
\end{aligned}
$$

where $1 / \widetilde{C}=1-1 / \beta$.

Applying Theorem 2.1, we have the following nice simple condition for (7).

Corollary 2.2 Suppose $\sigma_{t} \leq b<\pi^{2} / 2$, where $b$ is a positive constant. Then

$$
\|u\|_{x}^{2} \leq C a(u, u), \quad \forall u \in V,
$$

where $C$ is a positive constant only depending on $b$.

Proof. Since $b<\pi^{2} / 2$, there exists a positive constant $c$ such that $b<c<\pi^{2} / 2$. Consider the function $g(x)=\sqrt{2} b \tan \sqrt{\frac{c}{2}} x$. A simple computation shows that 


$$
\begin{aligned}
g_{x}-\sigma_{t} & \geq g_{x}-b=\sqrt{ } b c \tan ^{2} \sqrt{\frac{c}{2}} x+\sqrt{ } b(\sqrt{ } c-\sqrt{ } b) \\
& =\frac{1}{\sqrt{\frac{c}{b}}} g^{2}+\sqrt{ } b(\sqrt{ } c-\sqrt{ } b) .
\end{aligned}
$$

Applying Theorem 2.1 finishes the proof.

The following example shows that the difference between $\sigma_{t}<\pi^{2} / 2$ and Vanaja-Kellogg's assumption $\sigma_{t} \leq 0$ is essential.

Example 1. Let $\sigma=\mathrm{e}^{t} \theta(x)-\varphi(x)$, where $\theta(x)$ and $\varphi(x)$ are a continuous function for $-1 \leq x \leq 1$ such that $\sigma$ changes sign in $\Omega$ and $\varphi(x) \leq b<\pi^{2} / 2$. Doing a transformation $y=\left(1-\mathrm{e}^{-t}\right) /\left(1-\mathrm{e}^{-1}\right)$ for $(1),(2)$, we obtain a new forwardbackward heat equation

$$
\alpha(x, y) v_{y}-v_{x x}=\widetilde{f}(x, y), \quad \forall(x, y) \in(-1,1) \times(0,1)
$$

subject to condition (2), where $v(x, y)=u(x, t), \tilde{f}(x, y)=f(x, t)$ and $\alpha(x, y)=$ $(\theta(x)-\varphi(x)) /\left(1-\mathrm{e}^{-1}\right)+\varphi(x) y$. Therefore, $\alpha_{y}(x, y)=\varphi(x) \leq b<\pi^{2} / 2$.

Denote the corresponding $\sigma$ by $\alpha$ after transformation $y=y(t)$. Now we show that there is no transformation $y=y(t)$ such that $\sigma_{t} \leq 0$ in general. A straightforward computation shows

$$
\alpha_{y}=\sigma_{t}+\left(\log y^{\prime}\right)^{\prime} \sigma .
$$

Hence, for the zero points of $\sigma, \alpha_{y}=\sigma_{t}=\mathrm{e}^{t} \theta(x)=\varphi(x)$, which implies that there is no transformation $y=y(t)$ such that $\alpha_{t} \leq 0$ if $\varphi(x)>0$ on the zero points of $\sigma$.

Inequality (7) may not be true for some $\sigma$ if the conditions of Corollary 2.2 are not satisfied. The following example shows that there exists at least one function $u \in V$ such that $a(u, u) \leq 0$ if $\inf \sigma_{t} \geq \pi^{2} / 2$.

Example 2. Assume that inf $\sigma_{t} \geq \pi^{2} / 2$. Consider $u=t(t-1) \cos { }_{2}^{\pi} x$. It is readily seen that

$$
\begin{aligned}
a(u, u) & =\int_{\Omega}\left(-\frac{1}{2} \sigma_{t} u^{2}+u_{x}^{2}\right) d \Omega \leq \int_{\Omega}\left(-\frac{\pi^{2}}{4} u^{2}+u_{x}^{2}\right) d \Omega \\
& =-\frac{\pi^{2}}{4} \int_{\Omega} t(t-1)\left(\cos ^{2} \frac{\pi}{2} x-\sin ^{2} \frac{\pi}{2} x\right) d \Omega=0
\end{aligned}
$$

Example 2 shows that the Galerkin approximation based on (5) may fail for solving (1), (2) if the conditions of Theorem 2.1 are not satisfied. Our next example, however, shows that there exists a positive constant $C$ such that (7) hold for some $\sigma \in H^{1}(\Omega)$ even if $\sigma_{t} \geq \pi^{2} / 2$ for some points $(x, t) \in \Omega$.

Example 3. Let $\sigma=t x \tan ^{2} b x$, where $0<b<\pi / 2$ and $-1 \leq x \leq 1$. Consider $g(x)=c \tan d x$ with $\pi / 2>d>\max (\sqrt{ } 2, b)$ and $d-\sqrt{ } d^{2}-2<c<d+$ $\sqrt{ } d^{2}-2$. We have 


$$
\begin{aligned}
g_{x}-\sigma_{t} & =\frac{c d}{\cos ^{2} d x}-x \tan ^{2} b x \geq \frac{c d}{\cos ^{2} d x}-\tan ^{2} b x \\
& \geq \frac{c d}{\cos ^{2} d x}-\tan ^{2} d x \geq(c d-1) \tan ^{2} d x \geq \frac{\beta}{2} g^{2},
\end{aligned}
$$

where $\beta=2(c d-1) / c^{2}>1$. Theorem 2.1 shows that there exists a positive constant $C$ such that $a(u, u) \geq C\|u\|_{x}^{2}$ for $\forall u \in V$.

\section{A weighted Galerkin variational formulation}

As we have seen in the previous section, the Galerkin approximation fails for solving equations like $\sigma(x, t) u_{t}-u_{x x}=f(x, t)$ in some cases. In this section, we will introduce a weighted Galerkin variational formulation for (1), (2) to solve a wide class of the equations. To this end, let $w(x, t) \in H^{1}(\Omega)$ be a function such that $k_{1} \leq w(x, t) \leq k_{2}$, where $k_{1}$ and $k_{2}$ are positive constants, and $W(u, v)=\left(\sigma w u_{t}, v\right)+\left(w_{x} u_{x}, v\right)+\left(w u_{x}, v_{x}\right)$. Our weighted variational formulation of equation (1) is to find $u \in V$ such that

$$
W(u, v)=(w f, v), \quad \forall v \in V .
$$

Theorem 3.1 If there are two functions $g, q \in H^{1}(\Omega)$ such that $|q| \leq a<+\infty$ and

$$
\frac{1}{2} g_{x}-\frac{1}{2} \sigma_{t}-\sigma q_{t}-q_{x}^{2} \geq \frac{\beta}{4} g^{2}
$$

with $\beta>1$, then there exists a positive function $w \in H^{1}(\Omega)$ such that $k_{1} \leq w \leq k_{2}$ and

$$
W(u, u) \geq C\|u\|_{x, w}^{2} .
$$

where $k_{1}, k_{2}$ and $C$ are positive constants.

Proof. Consider $w=\alpha^{2}$, where $\alpha=\exp (q)$. Then

$$
W(u, u)=\int_{\Omega}\left(\sigma \alpha^{2} u_{t} u+2 \alpha_{x} \alpha u_{x} u+\alpha^{2} u_{x}^{2}\right) d \Omega .
$$

Since

$$
\begin{aligned}
& \alpha^{2} u_{t} u=(\alpha u)_{t}(\alpha u)-\frac{\alpha_{t}}{\alpha}(\alpha u)^{2}=\widetilde{u}_{t} \widetilde{u}-q_{t} \widetilde{u}^{2}, \\
& \alpha^{2} u_{x}^{2}=(\alpha u)_{x}^{2}-\alpha_{x}^{2} u^{2}-2 \alpha_{x} \alpha u_{x} u=\widetilde{u}_{x}^{2}-q_{x}^{2} \widetilde{u}^{2}-2 \alpha_{x} \alpha u_{x} u,
\end{aligned}
$$

where $\widetilde{u}=\alpha u$, it follows from the proof of Theorem 2.1 that

$$
\begin{aligned}
W(u, u) & =\int_{\Omega}\left(\sigma \widetilde{u}_{t} \widetilde{u}-\sigma q_{t} \widetilde{u}^{2}-q_{x}^{2} \widetilde{u}^{2}+\widetilde{u}_{x}^{2}\right) d \Omega \\
& \geq \int_{\Omega}\left(\left(\frac{1}{2} g_{x}-\frac{1}{2} \sigma_{t}-\sigma q_{t}-q_{x}^{2}\right) \widetilde{u}^{2}+g \widetilde{u}_{x} \widetilde{u}+\widetilde{u}_{x}^{2}\right) d \Omega .
\end{aligned}
$$

Hence, if (11) holds, we have (12). 
Theorem 3.1 and Corollary 2.2 show the following result.

Corollary 3.2 If there exists a function $q \in H^{1}(\Omega)$ such that $|q|<a<+\infty$ and

$$
\frac{1}{2} \sigma_{t}+\sigma q_{t}+q_{x}^{2} \leq b<\frac{\pi^{2}}{4},
$$

then there exist a positive function $w \in H^{1}(\Omega)$ such that $k_{1} \leq w \leq k_{2}$ and

$$
W(u, u) \geq C\|u\|_{x, w}^{2},
$$

where $k_{1}, k_{2}$ and $C$ are positive constants.

The following example shows that the conditions of Corollary 3.2 still hold for some $\sigma$ even if (7) fails.

Example 4. Consider $\sigma={ }_{2}^{1} x+b t^{2}$, where $\pi^{2} / 4 \leq b \leq \pi^{2}-1$.

First we prove that there is no function $g \in H^{1}(\Omega)$ such that (6) holds. If it is not the case, we have $g_{x} /\left(\beta g^{2} / 2+2 b t\right) \geq 1$, which implies that

$$
g(x, t) \geq 2 \sqrt{\frac{b t}{\beta}} \tan (\sqrt{ } b \beta t x+c(t)) .
$$

Since $g(x, t) \in H^{1}(\Omega)$ and $g(0, t) \geq \tan c(t)$, we have that $c(t)$ is bounded by

$$
m \pi-\frac{\pi}{2}<c(t)<m \pi+\frac{\pi}{2},
$$

where $m$ is an integer. Let $c(t)=m \pi+\delta(t)_{2}^{\pi}$ with $-1<\delta(t)<1$. (14) yields that

$$
g(x, t) \geq 2 \sqrt{\frac{b t}{\beta} \tan \left(\sqrt{ } b \beta t x+\delta(t) \frac{\pi}{2}\right) .}
$$

Since $b \geq \pi^{2} / 4$ and $\beta>1$, there are some points $\left(x_{0}, t_{0}\right) \in(-1,1] \times[0,1]$ such that

$$
\begin{aligned}
& \sqrt{ } b \beta t x+\delta(t) \frac{\pi}{2} \rightarrow \frac{\pi}{2}-0, \\
& \tan \left(\sqrt{ } b \beta t x+\delta(t) \frac{\pi}{2}\right) \rightarrow \frac{\pi}{2}-\sqrt{ } b \beta t x-\delta(t)_{2}^{\frac{\pi}{2}},
\end{aligned}
$$

when $(x, t) \rightarrow\left(x_{0}-0, t_{0}\right)$ or

$$
\begin{aligned}
& \sqrt{ } b \beta t x+\delta(t) \frac{\pi}{2} \rightarrow-\frac{\pi}{2}+0, \\
& \tan \left(\sqrt{ } b \beta t x+\delta(t) \frac{\pi}{2}\right) \rightarrow \frac{1}{-\frac{\pi}{2}-\sqrt{ } b \beta t x-\delta(t)_{2}^{\frac{\pi}{2}},}
\end{aligned}
$$

when $(x, t) \rightarrow\left(x_{0}+0, t_{0}\right)$. For example 


$$
\left(x_{0}, t_{0}\right)=\left\{\begin{array}{cc}
\left(\begin{array}{cc}
-\delta\left(\frac{1}{\beta}\right)_{2}^{\pi}+\frac{\pi}{2}, \frac{1}{\beta} \\
\sqrt{ } b
\end{array}\right. & \text { if } \delta\left(\begin{array}{l}
1 \\
\beta
\end{array}\right) \geq 0 \\
\left(\begin{array}{cc}
-\delta\left({ }_{\beta}^{1}\right)_{2}^{\pi}-\frac{\pi}{2} & 1 \\
\sqrt{ } b & \frac{\pi}{\beta}
\end{array}\right) & \text { if } \delta\left(\begin{array}{l}
1 \\
\beta
\end{array}\right)<0 .
\end{array}\right.
$$

This contradicts to that $g \in H^{1}(\Omega)$. In particular, it is easy to find a function $u \in V$ such that $a(u, u) \leq 0$ if $b \geq \pi^{2} / 2$. For example, $u=t(t-1) \cos { }_{2}^{\pi} x$.

Now we prove that there is a function $q \in H^{1}(\Omega)$ such that (13) holds. Consider $q=-\sigma^{2}$. A computation shows that

$$
\begin{aligned}
& \frac{1}{2} \sigma_{t}+\sigma q_{t}+q_{x}^{2}=b t-4 b t \sigma^{2}+\sigma^{2} \\
\leq & \sqrt{\frac{b}{2}}(2 \sigma+1)^{\frac{1}{2}}\left(1-4 \sigma^{2}\right)+\sigma^{2} .
\end{aligned}
$$

Note that $\sigma>-\frac{1}{2}$. Let $r(x)=\sqrt{\frac{b}{2}}(2 x+1)^{\frac{1}{2}}\left(1-4 x^{2}\right)+x^{2}$ with $x>-\frac{1}{2}$. Then

$$
r^{\prime}(x)=-\sqrt{\frac{b}{2}}(2 x+1)^{\frac{1}{2}}(10 x-1)+2 x .
$$

If $x \geq \frac{1}{2}, r^{\prime}(x) \leq-\sqrt{ } b(10 x-1)+2 x<0$. Hence

$$
\begin{aligned}
\max _{x>-\frac{1}{2}} r(x) & =\max _{-\frac{1}{2}<x<\frac{1}{2}} r(x) \leq \max _{-\frac{1}{2}<x<\frac{1}{2}} \sqrt{\frac{b}{2}(2 x+1)^{\frac{1}{2}}\left(1-4 x^{2}\right)+\frac{1}{4}} \\
& =\sqrt{\frac{b}{2}\left(2 \cdot 10^{-1}+1\right)^{\frac{1}{2}}\left(1-4 \cdot 10^{-2}\right)+\frac{1}{4}} \\
& \leq \sqrt{\frac{\pi^{2}-1}{2}\left(\begin{array}{c}
6 \\
10
\end{array}\right)^{\frac{1}{2}}\left(\begin{array}{l}
24 \\
25
\end{array}\right)+\frac{1}{4}} \\
& <\frac{\pi^{2}}{4}
\end{aligned}
$$

This example implies that the weighted method based on (10) can be useful for the solution of a wide class of the problems of the type $\sigma(x, t) u_{t}-u_{x x}=f(x, t)$ where $\sigma(x, t)$ changes sign in $\Omega$ in case the Galerkin method based on (5) fails for the purpose. Therefore, the weighted variational formulation (10) is an essential generalization of the variational formulation (5) for the equation (1), (2).

\section{Galerkin approximations and discretization error estimates}

In this section, we will discretize our finite element schemes and derive $L^{2}$ error estimates for the Galerkin approximations. The Galerkin variational formulation can be viewed as a special case $w=1$ of the weighted Galerkin variational formulation. 
Let $V^{h}$ be a finite-dimensional subspace of Hilbert space $V$ satisfying the boundary condition $\left.u\right|_{\Gamma}=0$, where $\Gamma=\Gamma_{2} \cup \Gamma_{3} \cup \Gamma_{5} \cup \Gamma_{6}$. The weighted Galerkin approximation of the equation (1) is to find a $u^{h} \in V^{h}$ such that

$$
W\left(u^{h}, v^{h}\right)=\left(w f, v^{h}\right), \quad \forall v^{h} \in V^{h} .
$$

Theorem 4.1 Assume the conditions of Theorem 3.1 hold. Then there exists a unique $u^{h} \in V^{h}$ satisfying (15). Moreover,

$$
\left\|u^{h}\right\|_{x, w} \leq C\|f\|_{0}
$$

where $C$ is a positive constant.

Proof. Let $\left\{\phi_{j}\right\}$ be a basis for $V^{h}$ and denote $u^{h}=\sum u_{j} \phi_{j}, \mathbf{u}=\left(u_{1}, \ldots, u_{n}\right)^{\mathrm{T}}$ and $\mathbf{b}=\left(b_{1}, \ldots, b_{n}\right)^{\mathrm{T}}$, where $b_{i}=\left(w f, \phi_{i}\right)$. Then $\mathbf{u}$ is the solution of the following linear system

$$
A \mathbf{u}=\mathbf{b},
$$

where $A=\left(a_{i j}\right)_{i, j=1}^{n}$ with $a_{i j}=W\left(\phi_{j}, \phi_{i}\right)$. It follows from (12) that $A$ is a positive definite matrix. Hence (17) has a unique solution. On the other hand, Theorem 3.1 shows

$$
\left\|u^{h}\right\|_{x, w}^{2} \leq C_{1} W\left(u^{h}, u^{h}\right)=C_{1}\left(w f, u^{h}\right) \leq C\|f\|_{0}\left\|u^{h}\right\|_{x, w},
$$

which implies (16).

It is shown in Lu and Wen (1994) that the inequality (16) holds for the canonical solution $u$ of (1), (2), i.e., $\|u\|_{x, w} \leq \widetilde{C}\|f\|_{0}$, where $\widetilde{C}$ is a positive constant. Therefore, both the canonical solution of (1), (2) and our finite element solution depend continuously on the right hand side function of the equation (1).

The linear system (17) of the discrete equations arising from the method is positive definite. We have a large number of efficient algorithms to solve it, for example, generalizations of the conjugate gradient method, e.g. Eisenstat, Elman and Schultz (1983), Saad (1981) showing monotone convergence for positive definite linear systems. One can also use sparse $L U$-factorization for the system.

We now derive $L^{2}$ error estimates for the Galerkin approximation (15).

Theorem 4.2 Let $u$ and $u^{h}$ be solutions of problems (10) and (15), respectively. If conditions of Theorem 3.1 hold, then there exists a positive constant $C$ such that

$$
\left\|u-u^{h}\right\|_{x, w} \leq C \inf _{v^{h} \in V^{h}}\left\|w\left(u-v^{h}\right)\right\|_{1} .
$$

Proof. For a given $v^{h} \in V^{h}$ Theorem 3.1 shows that

$$
\begin{aligned}
& C_{1}\left\|u^{h}-v^{h}\right\|_{x, w}^{2} \leq W\left(u^{h}-v^{h}, u^{h}-v^{h}\right) \\
= & W\left(u-v^{h}, u^{h}-v^{h}\right) \leq C_{2}\left\|w\left(u-v^{h}\right)\right\|_{1}\left\|u^{h}-v^{h}\right\|_{x, w} .
\end{aligned}
$$

Setting $C_{3}=C_{2} / C_{1}$ shows that $\left\|u^{h}-v^{h}\right\|_{x, w} \leq C_{3}\left\|w\left(u-v^{h}\right)\right\|_{1}$. Since

$$
\left\|u-u^{h}\right\|_{x, w} \leq\left\|w\left(u-v^{h}\right)\right\|_{1}+\left\|u^{h}-v^{h}\right\|_{x, w},
$$

choosing $C=C_{3}+1$ shows our result. 
To analyze the error of our method, we make the following assumptions:

1. There is an $s \geq 0$ such that $u \in V \cap H^{s}(\Omega)$.

2. $\left\{V^{h}\right\}_{h>0}$ is a regular family of finite elements, where $V^{h}$ is a subspace of $V$ consisting of piecewise polynomials of degree $k$ with $k \leq s-1$.

Now we have the error estimate as follows:

Theorem 4.3 If conditions of Theorem 3.1 and assumptions 1, 2 hold, then there exists a positive constant $C>0$ such that

$$
\left\|u-u^{h}\right\|_{x, w} \leq C h^{k}|u|_{k+1} .
$$

Proof. The theorem follows from Theorem 4.2 and the usual interpolation theoretic result.

Corollary 4.4 If conditions of Theorem 3.1 and assumptions 1, 2 hold, then there exists a positive constant $C>0$ such that

$$
\left\|u-u^{h}\right\|_{0} \leq C h^{k}|u|_{k+1}
$$

Proof. Since $w>k_{1}>0$,

$$
\left\|u-u^{h}\right\|_{0} \leq \frac{\left\|w\left(u-u^{h}\right)\right\|_{0}}{k_{1}} \leq \frac{\left\|u-u^{h}\right\|_{x, w}}{k_{1}} .
$$

The corollary follows from Theorem 4.3.

\section{Comparison with Aziz-Liu's method}

Aziz and Liu (1991) presented a finite element method for the problem (1), (3) by reducing the equation to a first order system of partial differential equations under the following assumptions

$$
\begin{aligned}
& H 1: \lambda \alpha \sigma-\frac{1}{2}(\alpha \sigma)_{t}+\frac{1}{2}(\gamma \sigma)_{x} \geq k_{1}, \\
& H 2: \alpha \geq k_{2}, \\
& H 3: \alpha_{x}+\gamma \sigma<2 \sqrt{ } k_{1} k_{2} \\
& H 4: \sigma n_{t} \mid \Upsilon_{1} \cup \Upsilon_{4} \geq 0,
\end{aligned}
$$

where $k_{1}>0$ and $k_{2}>0$ are constants, $\Upsilon_{1}=\{(x, 0):-1<x<0\}$ and $\Upsilon_{4}=\{(x, 1): 0<x<1\}$.

In general, we cannot guarantee the existence of a solution for the problem (1), (3) as it was pointed in Lu (1995) even if $H 1-H 4$ are satisfied. Fortunately, AzizLiu's method can be used to solve (1), (2) with a straightforward modification under $H 1-H 3$. In fact, by a transformation

$$
\mathbf{u}=\left(\begin{array}{l}
u_{1} \\
u_{2}
\end{array}\right), \quad u_{1}=\mathrm{e}^{-\lambda t} u, \quad u_{2}=\mathrm{e}^{-\lambda t} u_{x}
$$


it follows from Aziz and Liu (1991) that equation (1) may be written as the symmetric first-order system

$$
A_{1} \mathbf{u}_{x}+A_{2} \mathbf{u}_{t}+A_{3} \mathbf{u}=\mathbf{f}
$$

where

$$
A_{1}=\left(\begin{array}{cc}
0 & -1 \\
-1 & 0
\end{array}\right), A_{2}=\left(\begin{array}{ll}
\sigma & 0 \\
0 & 0
\end{array}\right), A_{3}=\left(\begin{array}{cc}
\lambda \sigma & 0 \\
0 & 1
\end{array}\right), \mathbf{f}=\left(\begin{array}{c}
\mathrm{e}^{-\lambda t} f \\
0
\end{array}\right) .
$$

Because of this, to solve the forward-backward equation Aziz and Liu presented a Galerkin method to find a vector-valued function $\mathbf{u}=\left(u_{1}, u_{2}\right)^{\mathrm{T}}: \Omega \rightarrow \mathbb{R}^{2}$, which is a solution of the first-order system

$$
A_{1} \mathbf{u}_{x}+A_{2} \mathbf{u}_{t}+A_{3} \mathbf{u}=\mathbf{g} \text { in } \Omega
$$

with the boundary condition $M \mathbf{u} \equiv u_{1}=0$ on $\Gamma_{2} \cup \Gamma_{3} \cup \Gamma_{5} \cup \Gamma_{6}$, where the function $\mathbf{g}=\left(f_{1}, f_{2}\right)^{\mathrm{T}} \in\left(L^{2}(\Omega)\right)^{2}$.

Define a $2 \times 2$ matrix-valued function $T$ by $T \mathbf{v}=\left(\begin{array}{cc}\alpha & 0 \\ \gamma \sigma & \alpha\end{array}\right) \mathbf{v}$, where $\alpha$ and $\gamma$ are known function to be specified such that $T$ is bounded, and define a function space $V$ by $V=\left\{\mathbf{u} \in\left(H^{1}(\Omega)\right)^{2}: M \mathbf{u}=0\right\}$. Let $B: V \times V \rightarrow \mathbb{R}$ by $B(\mathbf{u}, \mathbf{v})=\langle L \mathbf{u}, T \mathbf{v}\rangle$, where $\langle\cdot, \cdot\rangle$ denotes the $\left(L^{2}(\Omega)\right)^{2}$ inner product. Their weak formulation of (21) for a given $\mathbf{g} \in\left(L^{2}(\Omega)\right)^{2}$ is: to find a $\mathbf{u} \in V$ such that

$$
B(\mathbf{u}, \mathbf{v})=\langle\mathbf{g}, T \mathbf{v}\rangle, \quad \forall \mathbf{v} \in V .
$$

If $H 1-H 3$ hold, following Aziz and Liu (1991) shows that Aziz-Liu's fundamental result that there exists a positive constant $C$ such that the basic condition

$$
\|\mathbf{u}\|_{0}^{2} \leq C B(\mathbf{u}, \mathbf{u})
$$

holds for problem (1), (2).

First we show that if $H 1-H 3$ hold then there exist two functions $q$ and $g$ such that (11) holds. If follows from $H 3$ that there is a positive constant $\beta>1$ such that $\beta(\gamma \alpha)^{2} \leq 4 k_{1} k_{2}-2 \gamma \sigma \alpha_{x}-\alpha_{x}^{2}$. Let $q={ }_{2}^{1} \log \alpha-\lambda t$ and $g=\gamma \sigma / \alpha$. A straightforward computation shows that

$$
\begin{aligned}
& \frac{1}{2} g_{x}-\frac{1}{2} \sigma_{t}-\sigma q_{t}-q_{x}^{2} \\
= & \frac{1}{2}\left(\frac{(\gamma \sigma)_{x}}{\alpha}-\frac{\gamma \sigma \alpha_{x}}{\alpha^{2}}\right)-\frac{1}{2} \sigma_{t}-\frac{1 \sigma \alpha_{t}}{2 \alpha}+\lambda \sigma-\frac{1 \alpha_{x}^{2}}{4 \alpha^{2}} \\
= & \frac{\lambda \alpha \sigma-\frac{1}{2}(\alpha \sigma)_{t}+\frac{1}{2}(\gamma \sigma)_{x}}{\alpha}-\frac{2 \gamma \sigma \alpha_{x}+\alpha_{x}^{2}}{4 \alpha^{2}} \\
\geq & \frac{k_{1}}{\alpha}-\frac{k_{1} k_{2}}{\alpha^{2}}+\frac{\beta(\gamma \alpha)^{2}}{4} \alpha^{2} \geq \frac{\beta}{4} g^{2} .
\end{aligned}
$$

Second, let $u_{1}=\mathrm{e}^{-\lambda t} u, u_{2}=\mathrm{e}^{-\lambda t} u_{x}$. Then $u_{1_{x}}=u_{2}$. If $\mathbf{v}=(v, 0)^{\mathrm{T}}$, where $v \in V$, we have 


$$
\begin{aligned}
B(\mathbf{u}, \mathbf{v}) & =\int_{\Omega}\left(\sigma \alpha u_{1_{t}} v-\alpha u_{2_{x}} v+\lambda \sigma \alpha u_{1} v-\beta \sigma u_{1_{x}} v+\beta \sigma u_{2} v\right) d \Omega \\
& =\int_{\Omega}\left(\alpha \sigma u_{1_{t}} v+\lambda \sigma \alpha u_{1} v-\alpha u_{2_{x}} v\right) d \Omega \\
& =\int_{\Omega}\left(\sigma \alpha \mathrm{e}^{-\lambda t}\left(-\lambda u+u_{t}\right) v+\lambda \sigma \alpha \mathrm{e}^{-\lambda t} u v-\alpha \mathrm{e}^{-\lambda t} u_{x x} v\right) d \Omega \\
& =\int_{\Omega}\left(\sigma \alpha \mathrm{e}^{-\lambda t} u_{t} v-\alpha \mathrm{e}^{-\lambda t} u_{x x} v\right) d \Omega \\
& =\int_{\Omega}\left(\sigma \alpha \mathrm{e}^{-\lambda t} u_{t} v+\alpha_{x} \mathrm{e}^{-\lambda t} u_{x} v+\alpha \mathrm{e}^{-\lambda t} u_{x} v_{x}\right) d \Omega-\int_{\partial \Omega} \sigma \mathrm{e}^{-\lambda t} n_{x} u_{x} v d S \\
& =\int_{\Omega}\left(\sigma \alpha \mathrm{e}^{-\lambda t} u_{t} v+\alpha_{x} \mathrm{e}^{-\lambda t} u_{x} v+\alpha \mathrm{e}^{-\lambda t} u_{x} v_{x}\right) d \Omega
\end{aligned}
$$

In this case, choosing $w=\mathrm{e}^{-\lambda t} \alpha$, we have

$$
B(\mathbf{u}, \mathbf{v})=W(u, v), \quad \forall u \in V \cap H^{2}(\Omega) \text { and } v \in V,
$$

which implies that if Aziz and Liu's method is applicable to solve (1), (2), so is the weighted Galerkin method presented here. On the other hand, the weighted Galerkin method needs less computations than Aziz-Liu's method simply because the weighted Galerkin approximation is done on $L^{2}(\Omega)$ while the later one is on $\left(L^{2}(\Omega)\right)^{2}$. If the weak formulation (22) is used to solve the forward-backward heat equation (1), (2), it requires that $u, u_{x}, u_{t}, u_{t x}, u_{x x} \in L^{2}(\Omega)$. Our variational formulations (5) and (10) need $u \in H^{1}(\Omega)$. Finally, both methods share the same rate of convergence if they converge for (1), (2) in theory.

\section{Numerical examples}

In this section we implement our methods for some particular examples. We performed all of the following experiments by using triangular elements and piecewise linear functions as our basis. First, we consider the example implemented in Aziz and Liu (1991).

Example 5. Consider the problem (1), (2) with $\sigma(x, t)=x$ and

$$
f(x, t)=\left\{\begin{array}{cc}
2 x\left(x^{2}-1\right) t\left[(t-1)^{2}-4 x^{2}+t(t-1)\right] & \\
-2 t^{2}\left[(t-1)^{2}-24 x^{2}+4\right], & \forall x \geq 0, t \in[0,1], \\
2 x\left(x^{2}-1\right)(t-1)\left(2 t^{2}-t-4 x^{2}\right) & \\
-2(t-1)^{2}\left(t^{2}-24 x^{2}+4\right), & \forall x<0, t \in[0,1],
\end{array}\right.
$$

where $f$ has been chosen so that

$$
u(x, t)= \begin{cases}\left(x^{2}-1\right) t^{2}\left[(t-1)^{2}-4 x^{2}\right], & \forall x \geq 0, t \in[0,1], \\ \left(x^{2}-1\right)\left(t^{2}-4 x^{2}\right)(t-1)^{2}, & \forall x<0, t \in[0,1] .\end{cases}
$$


For this example we have

$$
\begin{aligned}
& \Gamma_{1}=\{(x, t): x \in(-1,0), t=0\}, \\
& \Gamma_{2}=\{(x, t): x=-1, t \in(0,1)\}, \\
& \Gamma_{3}=\{(x, t): x \in(-1,0), t=1\}, \\
& \Gamma_{4}=\{(x, t): x \in(0,1), t=1\}, \\
& \Gamma_{5}=\{(x, t): x=1, t \in(0,1)\}, \\
& \Gamma_{6}=\{(x, t): x \in(0,1), t=0\} .
\end{aligned}
$$

\begin{tabular}{|c|c|c|c|}
\hline & $\max |e|$ & $L^{2}$ error & $L^{2}$ rate \\
\hline & 0.5180 & 0.2456 & 1.4340 \\
\hline & 0.3237 & 0.0909 & \\
\hline & 0.1480 & 0.0274 & \\
\hline & 0.0849 & 0.0125 & 1.9356 \\
\hline & 0.0548 & 0.0069 & 2.0655 \\
\hline & 0.0285 & 0.0028 & 2.2244 \\
\hline & 0.0176 & 0.0015 & 2.1696 \\
\hline
\end{tabular}

In Table 1 we give some numerical results that show the performance of the Galerkin method.

Table 1. Computation of Galerkin method with $\sigma(x, t)=x$

Let $u$ be the solution of (1), (2) and $u^{h}$ be the solution of (15). The $L^{2}$ error in the Table 1 and throughout this section is defined by

$$
L^{2} \text { error }=\left\|u-u^{h}\right\|_{0}=\left(\int_{\Omega}\left|u-u^{h}\right|^{2} d \Omega\right)^{\frac{1}{2}} .
$$

Subdividing $\Omega$ into squares, Aziz and Liu (1991) performed their method with piecewise bivariate polynomials with degree $\leq 2$ as basis. Table 2 shows their numerical results.

Table 2. Computation of Aziz-Liu's method with $\sigma(x, t)=x$

\begin{tabular}{|c|c|c|c|}
\hline$h$ & $\max |e|$ & $L^{2}$ error & $L^{2}$ rate \\
\hline 1 & 4.271 & 1.104 & \\
\hline 2 & 1.208 & 0.276 & 1.99 \\
\hline 1 & 1.02 \\
\hline 1 & 0.316 & 0.067 & 2.02 \\
\hline 1 & 0.078 & 0.016 & 2.01 \\
\hline 16 & &
\end{tabular}

In Table 1 and Table 2, we can see the $L^{2}$ error and the $L^{2}$ rate of convergence for various meshsize $h$ of the Galerkin method and Aziz-Liu's method, respectively. The corresponding $\max |e|$ and the $L^{2}$ error in Table 1 are smaller than that in Table 2 . The corresponding $L^{2}$ rate becomes approximately the same after $h=1 / 8$. 
The second numerical example gives some numerical results when our method is applied to the problem (1), (2) with $\sigma_{t}(x, t)>\pi^{2} / 2$ in some points of $\Omega$.

Example 6. Let $\sigma(x, t)=x \exp \left((1-x)^{2}\right)+t \tan ^{2} \frac{9 \pi}{20} x$ and

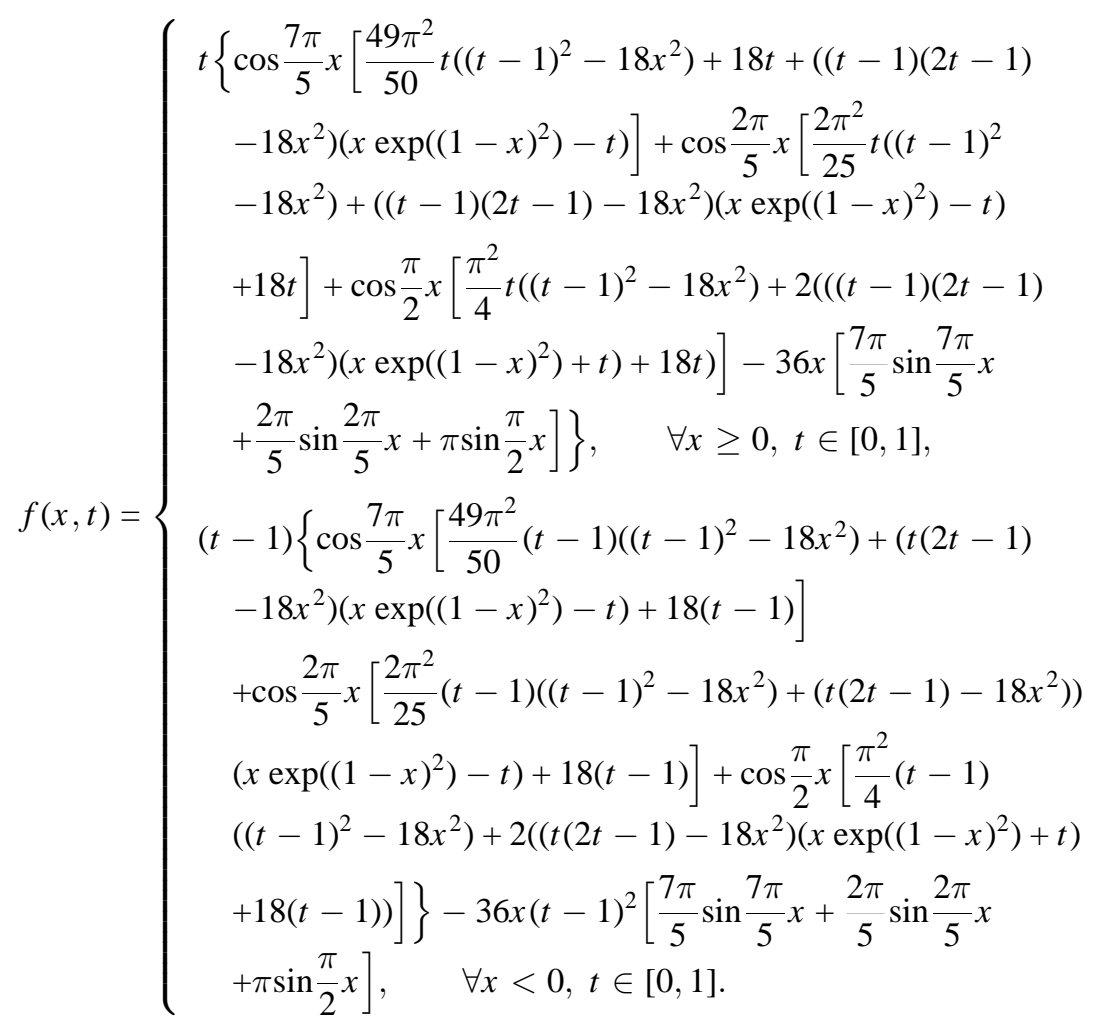

One can check the solution of the equation is given by

$$
u(x, t)=\left\{\begin{array}{cl}
\cos \frac{\pi}{2} x\left(\cos \frac{9 \pi}{10} x+1\right) t^{2}\left[(t-1)^{2}-18 x^{2}\right], & \forall x \geq 0, t \in[0,1], \\
\cos \frac{\pi}{2} x\left(\cos \frac{9 \pi}{10} x+1\right)(t-1)^{2}\left[t^{2}-18 x^{2}\right], & \forall x<0, t \in[0,1],
\end{array}\right.
$$

It follows from Example 3 that there is a function $g \in H^{1}(\Omega)$ such that $g_{x}-\sigma_{t} \geq{ }_{2}^{\beta} g^{2}$ with $\beta>1$ for $\sigma=x \exp \left((1-x)^{2}\right)+t \tan ^{2}{ }_{20}^{9 \pi} x$. Thus, the Galerkin method is available to this example. $\Gamma_{1}, \ldots, \Gamma_{6}$ are the same as those in Example 5. The numerical results in Table 3 show that the $L^{2}$ rate is approximately 2 .

Finally, the following example shows that the weighted Galerkin method can be used to solve (1), (2) efficiently even if the Galerkin method fails for the purpose.

Example 7. Consider the equation (1), (2) with $\sigma(x, t)={ }_{2}^{1} x+\frac{\pi^{2}+1}{2} t^{2}$ and 
Table 3. Computation of Galerkin Method with $\sigma=x \exp \left((1-x)^{2}\right)+t \tan ^{2}{ }_{20}^{9 \pi} x$

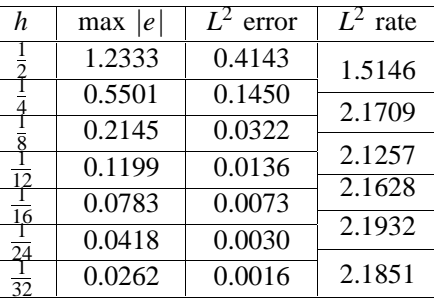

$$
\begin{aligned}
& f(x, t)=\left\{\begin{array}{c}
t \cos 2 \pi x\left\{[ x + ( \pi ^ { 2 } + 1 ) t ^ { 2 } ] \left[(t-1)(2 t-1)-8\left(\sqrt{ } x^{2}+1-1\right)\right.\right. \\
\left.\exp \left(\sqrt{ } x^{2}+1\right)\right]+\frac{\pi^{2} t}{4}\left[(t-1)^{2}-8\left(\sqrt{ } x^{2}+1-1\right)\right. \\
\left.\left.\exp \left(\sqrt{ } x^{2}+1\right)\right]+8 t\left[\frac{x^{2}}{\sqrt{ } x^{2}+1}+1\right] \exp \left(\sqrt{ } x^{2}+1\right)\right\} \\
-8 \pi t^{2} x \sin \frac{\pi x}{2} \exp \left(\sqrt{ } x^{2}+1\right), \quad \forall x \geq 0, t \in[0,1], \\
(t-1) \cos \frac{2 \pi x}{2}\left\{[ x + ( \pi ^ { 2 } + 1 ) t ^ { 2 } ] \left[t(2 t-1)-8\left(\sqrt{ } x^{2}+1-1\right)\right.\right. \\
\left.\exp \left(\sqrt{ } x^{2}+1\right)\right]+\frac{\pi^{2}(t-1)}{4}\left[t^{2}-8\left(\sqrt{ } x^{2}+1-1\right)\right. \\
\left.\left.\exp \left(\sqrt{ } x^{2}+1\right)\right]+8(t-1)\left[\frac{x^{2}}{\sqrt{ } x^{2}+1}+1\right] \exp \left(\sqrt{ } x^{2}+1\right)\right\} \\
-8 \pi t^{2} x \sin \frac{\pi x}{2} \exp \left(\sqrt{ } x^{2}+1\right), \quad \forall x<0, t \in[0,1] .
\end{array}\right.
\end{aligned}
$$

The solution of the equation is given by

$$
u(x, t)=\left\{\begin{array}{cc}
t^{2} \cos \frac{\pi x}{2}\left[(t-1)^{2}-8\left(\sqrt{ } x^{2}+1-1\right)\right. \\
\left.\exp \left(\sqrt{ } x^{2}+1\right)\right], & \forall x \geq 0, t \in[0,1], \\
(t-1)^{2} \cos \frac{\pi x}{2}\left[t^{2}-8\left(\sqrt{ } x^{2}+1-1\right)\right. \\
\left.\exp \left(\sqrt{ } x^{2}+1\right)\right], & \forall x<0, t \in[0,1] .
\end{array}\right.
$$

In this case the Galerkin method may fail when it is used to solve (1), (2) as mentioned in Example 4. Now we apply the weighted Galerkin method to solve it. To make the weighted method efficiently, we will seek a function $q(x, t) \in H^{1}(\Omega)$ such that

$$
{ }_{2}^{1} \sigma_{t}+\sigma q_{t}+q_{x}^{2}<\frac{\pi^{2}}{4}
$$

and the weight $w=\exp (2 q)$ is sufficient large so that the coefficient matrix of (17) is not near singular. To this end, let $q=-\frac{\sigma^{2}}{16}$. Similarly to Example 4, we find 


$$
\begin{aligned}
& \frac{1}{2} \sigma_{t}+\sigma q_{t}+q_{x}^{2}=\frac{\pi^{2}+1}{2} t-\frac{\pi^{2}+1}{8} t \sigma^{2}+\frac{\sigma^{2}}{256} \\
\leq & \max _{-1 / 2 \leq x \leq 2}\left(\sqrt{\left.\frac{\pi^{2}+1}{4}(2 x+1)\left(1-\frac{x^{2}}{4}\right)+\frac{x^{2}}{256}\right)}\right. \\
\leq & \sqrt{\frac{\pi^{2}+1}{20}(2 \sqrt{ } 21+3)\left(1-\frac{(\sqrt{ } 21-1)^{2}}{100}\right)+\frac{1}{64}} \\
< & \pi^{2} .
\end{aligned}
$$

For $\sigma={ }_{2}^{1} x+\frac{\pi^{2}+1}{2} t^{2}$ it is easy to see that $\Gamma_{1}, \Gamma_{2}, \Gamma_{5}, \Gamma_{6}$ are the same as those in Example 5, but $\Gamma_{3}=\emptyset$ and $\Gamma_{4}=\{(x, 1): x \in(-1,1)\}$. In Table 4, we give some

\begin{tabular}{|c|c|c|c|}
\hline & $\max |e|$ & $L^{2}$ error & $L^{2}$ rate \\
\hline & 1.6825 & 1.0988 & 1.2847 \\
\hline & 1.0249 & 0.4510 & \\
\hline & 0.5262 & 0.1494 & \\
\hline & 0.3233 & 0.0736 & \\
\hline & 0.2202 & 0.0441 & \\
\hline & 0.1223 & 0.0212 & 1.8065 \\
\hline & 0.0781 & 0.0125 & 1.8363 \\
\hline
\end{tabular}
numerical results that show the performance of the weighted Galerkin method for our last example.

Table 4. Computation of weighted Galerkin method with $\sigma={ }_{2}^{1} x+\frac{\pi^{2}+1}{2} t^{2}$

Acknowledgement. I am grateful to Professor O. Axelsson for valuable comments on the manuscript.

\section{References}

1. Aziz, A.K., Liu, J.-L. (1991): A Galerkin method for the forward-backward heat equation. Math. Comp. 56, 35-44

2. Baouendi, M.S., Grisvard, P. (1968): Sur une équation d'évolution changeant de type. J. Funct. Anal. 2, 352-367

3. Eisenstat, S.C., Elman, H.C., Schultz, M.H.(1983): Variational iterative methods for nonsymmetric systems of linear equations. SIAM J. Numer. Anal. 20, 345-357

4. Franklin, J.A., Rodemich, E.R. (1968): Numerical analysis of an elliptic-parabolic partial differential equation. SIAM J. Numer. Anal. 5, 680-716

5. Friedrichs, K.O. (1958): Symmetric positive differential equations. Comm. Pure Appl. Math. 11, $333-418$

6. Gevrey, M. (1913): Sur les équations aux dérivées partielles du type parabolique. J. Math. Pures Appl. 6, 305-475

7. Gevrey, M. (1914): Sur les équations aux dérivées partielles du type parabolique (suite). J. Math. pures Appl. 6, 105-148

8. LaRosa, T. (1986): The propagation of an electron beam through the solar corona. $\mathrm{PhD}$ thesis, Department of Physics and Astronomy, University of Maryland 
9. Lions, J. (1969): Quelques méthodes de résolution des problèmes aux limites non linéaires. Dunod Gauthier-Villars, Paris

10. Lu, H. (1995): Forward-backward heat equations and analysis of iterative methods. PhD thesis, Department of Mathematics, University of Nijmegen, The Netherlands

11. Lu, H., Wen Z.-Y. (1994): Solution of a forward-backward heat equation. Technical report, 9439, Department of Mathematics, University of Nijmegen, The Netherlands

12. Pagani, C.M. (1976): On forward-backward parabolic equations in bounded domains. Bolletino U. M. I. (5) 13-B, 336-354

13. Saad, Y. (1981): Krylov subspace methods for solving large unsymmetric linear systems. Math. Comp. 62, 105-126

14. Stewartson, K. (1974): Multistructural boundary layers on flat plates and related bodies. Adv. in Appl. Mech. 14, 145-239

15. Stewartson, K. (1981): D'Alembert's paradox. SIAM Rev. 23, 308-343

16. Vanaja, V., Kellogg, R.B. (1990): Iterative methods for a forward-backward heat equation. SIAM J. Numer. Anal. 27, 622-635

This article was processed by the author using the $\mathrm{L}_{\mathrm{E}} \mathrm{X}$ style file pljourlm from Springer-Verlag. 